\title{
Perforated Jejunal Diverticulosis: Report of a Case and Review of the Literature
}

\author{
Panagiotis Giannopoulos ${ }^{\mathrm{a}, \mathrm{c}}$, Dimitrios Linardoutsos ${ }^{\mathrm{b}}$, Tsamis Dimitrios ${ }^{\mathrm{a}}$, \\ Panagiotis Spirakopoulos $^{\mathrm{a}}$, Eirini Sklika ${ }^{\mathrm{a}}$, Georgios Patrinios ${ }^{\mathrm{a}}$
}

\begin{abstract}
Diverticular disease of the Jejunum is a rare entity. Most patients with small bowel diverticula are asymptomatic. Patients who develop symptoms generally report symptoms that reflect associated complications. The most common are nonspecific epigastric pain or flatulent dyspepsia, mainly post prandial. Complication rates as high as $10-12 \%$ for duodenal diverticulosis and $46 \%$ for jejunal diverticulosis have been reported. Although there is no consensus on the management of asymptomatic jejunal diverticula disease, some complications are potentially life-threatening and require early surgical intervention.
\end{abstract}

Keywords: Jejunal; Diverticulosis; Diverticulitis; Abscess; Perforation; Peritonitis; Enterectomy; Mortality

\section{Introduction}

Jejunal diverticulosis is uncommon amongst the overall diverticula occurring from the stomach to the rectosigmoid. They tend to be large and multiple and pathologically belong to the pseudodiverticula, resulting from a mucosal and submucosal herniation through the muscular layer of the bowels' wall in places of minor resistance to the intraluminal pressure such as the anatomic points where blood vessels penetrate the intestinal wall. They are generally asymptomatic but complications often occur as age advances with changes in the anatomy and physiology of the affected area. A wide spectrum of symptoms appear from mild epigastric or peri-umbilical discomfort, anaemia, bacterial overgrowth

\footnotetext{
Manuscript accepted for publication April 26, 2013

${ }^{a}$ Department of General Surgery, Kalamata General Hospital, Greece

${ }^{b} 1$ st Department of Propaedeutic Surgery, Hippocration General Hospital, Athens Medical School, University of Athens, Greece ${ }^{\mathrm{c}}$ Corresponding author: Patrinios Georgios, 35 Fellows road, London, NW3 3DX, United Kingdom. Email: gpatrin@gmail.com
}

doi: http://dx.doi.org/10.4021/jmc1276w with flatulence, malabsorption syndrome, fatigue and weight loss, to more serious complications such as diverticulitis, acute inflammation, intestinal obstruction and perforation with localized abscess causing abdominal pain with or without signs of peritonitis.

The management of jejunal diverticulosis with perforated diverticulitis usually requires surgical referral. When the patient are stable, trial of nonsurgical management with intravenous antibiotics and other supportive measures alongside percutaneous CT-guided aspiration of localised intraperitoneal collections maybe suitable and avoid the need for laparotomy. The disease should always be taken in consideration in the presence of non specific GI symptoms, especially in the elderly population. We herein report the case of a 63-year-old Caucasian man presenting with one-day history of generalised abdominal pain, on a background of a chronic abdominal discomfort, pain and dyspepsia. Our patient underwent a laparotomy which revealed multiple small jejunal diverticula and two larger adjacent diverticula arising on the mesenteric border of the proximal jejunum. Both diverticula were inflamed and perforated. We also review the literature for this rare disease.

\section{Case Report}

A 63-year-old Caucasian man arrived at the emergency department with one-day history of generalised abdominal pain on a background of a chronic abdominal discomfort, dyspepsia, pain, flatulence and borborygmi. No significant past medical history was noticed. OGD endoscopy and colonoscopy performed in the last two years were clear. The patient was admitted with right-side abdominal pain, localised at the lower right quadrant, rebound tenderness, pyrexia, significantly raised white cell count, inflammatory markers hypoalbuminemia and a raise to serum amylase. Chest and abdominal X-rays displayed only a few small intestinal air-fluid levels but not pneumoperitoneum. The patient was taken to the operating theatre on the first 24 hours considering appendicitis. A right paramedian incision exploratory laparotomy was performed which identified several small jejunal diverticula and two larger adjacent diverticula arising 


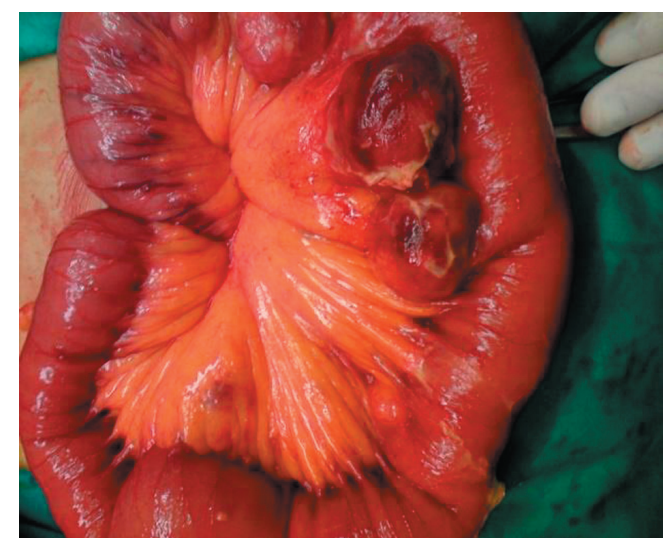

Figure 1. Intraoperative findings. Multiple inflamed diverticula arising at the mesenteric border of the jejunum.

on the mesenteric border of the proximal jejunum (Fig. 1). Both diverticula were inflammed and perforated containing a collection of pus (Fig. 2). A careful proximal and distal to the diverticula bowel mobilisation was performed, followed by a resection of the affected segment, using a gastrointestinal anastomosis stapling device. The rest of the diverticula were preserved. A side to side jejuno-jejunal stapled anastomosis was created, followed by a copious peritoneal lavage. A Jackson-Pratt drain was left near the anastomosis. Pathology report described large diverticula and multiple foci of diverticulitis with perforations. The patient did well postoperatively and was discharged on the sixth day.

\section{Discussion}

The disease was first described by Sommering in 1794 and later by Astley Cooper in 1809. Gordinier and Shil performed the first operation for diverticula in 1906 [1,2]. The incidence of jejunoileal diverticula in studies of the small bowel by enteroclysis is $2-2.3 \%$ which is comparable to autopsy data presenting an incidence of 1.3-4.6\% [3, 4] Incidence in radiographic findings is $2.3 \%$ [5]. Duodenal diverticula are approximately 5 times more common than jejunoileal diverticula. The prevalence increases with the age and the disease presents a peak incidence at the sixth and seventh decades with a male predominance [6].

The jejunal diverticulosis is usually multiple and probably present due to the larger size of the vasa recta at this area [4]. The aetiology is unclear. Studies have focused on abnormalities of the smooth muscles or of the myenteric plexus in order to explain intestinal dyskinesia. Manometric studies in such patients have also shown functional abnormalities. The results support the hypothesis that irregular intestinal contractions generate increased segmental intraluminal pressure, favouring the diverticula formation through the weakest point of the bowel $[7,8]$. Despite small bowel

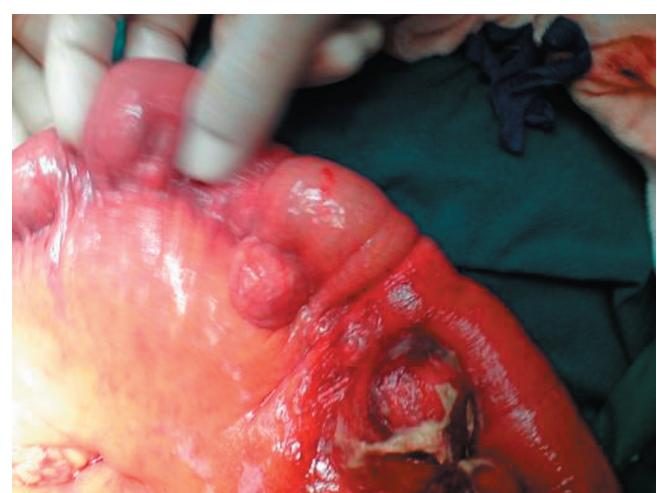

Figure 2. Intraoperative findings. Perforated diverticulum arising at the mesenteric border of the jejunum.

diverticulosis seems to be acquired, two cases of familiar predisposition have been reported $[9,10]$. The majority of patients are asymptomatic for life. However 30\% develop symptoms, experiencing epigastric pain, abdominal discomfort, post prandial flatulence, epigastric cramping pain and fever are usually reported [3]. Anemia due to chronic occult gastrointestinal bleeding, iron or B12 deficiency has often been reported and commonly attributed to malabsorption syndrome with diarrhoea and steatorrhea. Malabsorption could be justified by the nonsynchronous peristaltic movement of the bowel, the dilation of the diverticula, the stasis of the intestinal content and the bacterial overgrowth [11].

Considering that jejunal diverticulosis is asymptomatic for a long time in most of the cases, diagnosis is usually made when the disease becomes symptomatic or complicated. The diagnosis is often missed or delayed. It is a challenging disorder from a diagnostic perspective, with no truly reliable diagnostic tests. The literature has shown that computer tomography scan has variable reliability, while barium swallows are the gold standard for diagnosis [12]. Computer tomography may identify localized intestinal wall thickening due to inflammation or edema, abscesses, free abdominal fluids and pneumoperitoneum. Multislice CT seems to be promising in diagnosing jejunal diverticulosis and appears more specific than enteroclysis concerning small bowel diseases and may identify the enterolith inside the diverticulum or protruding into the intestinal lumen [13]. Endoscopic procedures, such as single- or double-balloon enteroscopy and wireless capsule endoscopy, are useful in diagnosing small bowel disorders, however these procedures are expensive, present low availability and cannot be used in the emergency setting, such as intestinal obstruction or perforation $[14,15]$.

Complications of jejunal diverticulosis warranting surgical intervention occur in $10-30 \%$ of patients and the most common acute complications include diverticulitis, bleeding, enterolith formation, intestinal obstruction and perforation [16-18], however, some patients respond to the temporary interruption of the enteral nutrition, to a gastrointestinal 
relief with a nasogastric tube and to the administration of empirical, wide-spectrum antibiotics [19, 20]. Another series of perforated diverticulitis treated conservatively with antibiotic administration and CT-guided drainage of abdominal abscesses have also been presented [21]. Instigating factors for perforation were shown to be related to a necrotizing inflammatory reaction in $82 \%$ of cases, followed by blunt trauma (12\%) and foreign body impaction (6\%). Cocaine sniffing has also been reported as a risk factor [22, 23]. The incidence of jejunal diverticulitis with or without perforation ranges from $2-6 \%$ [24].

The current treatment of choice for perforated jejunal diverticula causing peritonitis is exploratory laparotomy and resection of affected intestinal segment with primary anastomosis. The extent of the bowel resection depends upon the length of the bowel that is affected by the diverticula and the patient's peri-operative condition [25]. If diverticula are extensive, resection may have to be limited to include only the segment containing the perforated diverticulum and to leave a segment of small bowel that still contains non-perforated diverticula in order to avoid short bowel syndrome [26].

Recently, the total laparoscopic approach has been used as a valid surgical strategy, even in cases of complicated diverticulitis [27]. Laparoscopy becomes a valid diagnostic approach as it is rapidly convertible in laparotomy and it can function as a guide in order to avoid unnecessary laparotomies [28]. Our case in this paper presented symptomatology mimicking acute appendicitis and a decision was taken to perform prompt exploratory laparotomy due to the extent of the clinical and haematological findings and the individual Surgeon's experience.

\section{Conclusions}

It is seen that complicated jejunal diverticula are quite rare and that one of the most common complication is perforation. The treatment of choice is surgical excision of the affected jejunal segment. However, conservative management has been reported in selected patients to alleviate symptoms and inflammation before proceeding to an elective resection and primary anastomosis. If affected diverticula are not resected complications will reoccur. It is important to be familiar with this entity and take it in consideration in cases of unexplained gastrointestinal symptoms, because any treatment delay may contribute to an unsuccessful clinical outcome for patients.

\section{References}

1. Longo WE, Vernava AM, 3rd. Clinical implications of jejunoileal diverticular disease. Dis Colon Rectum. 1992;35(4):381-388.

2. Williams RA, Davidson DD, Serota AI, Wilson SE. Sur- gical problems of diverticula of the small intestine. Surg Gynecol Obstet. 1981;152(5):621-626.

3. Maglinte DD, Chernish SM, DeWeese R, Kelvin FM, Brunelle RL. Acquired jejunoileal diverticular disease: subject review. Radiology. 1986;158(3):577-580.

4. Ross CB, Richards WO, Sharp KW, Bertram PD, Schaper PW. Diverticular disease of the jejunum and its complications. Am Surg. 1990;56(5):319-324.

5. Kassahun WT, Fangmann J, Harms J, Bartels M, Hauss J. Complicated small-bowel diverticulosis: a case report and review of the literature. World J Gastroenterol. 2007;13(15):2240-2242.

6. Lempinen M, Salmela K, Kemppainen E. Jejunal diverticulosis: a potentially dangerous entity. Scand J Gastroenterol. 2004;39(9):905-909.

7. Krishnamurthy S, Kelly MM, Rohrmann CA, Schuffler MD. Jejunal diverticulosis. A heterogenous disorder caused by a variety of abnormalities of smooth muscle or myenteric plexus. Gastroenterology. 1983;85(3):538547.

8. Kongara KR, Soffer EE. Intestinal motility in small bowel diverticulosis: a case report and review of the literature. J Clin Gastroenterol. 2000;30(1):84-86.

9. Koch AD, Schoon EJ. Extensive jejunal diverticulosis in a family, a matter of inheritance? Neth J Med. 2007;65(4):154-155.

10. Andersen LP, Schjoldager B, Halver B. Jejunal diverticulosis in a family. Scand J Gastroenterol. 1988;23(6):672674.

11. Balducci G, Dente M, Cosenza G, Mercantini P, Salvi PF. Multiple giant diverticula of the foregut causing upper gastrointestinal obstruction. World J Gastroenterol. 2008;14(20):3259-3261.

12. Chugay P, Choi J, Dong XD. Jejunal diverticular disease complicated by enteroliths: Report of two different presentations. World J Gastrointest Surg. 2010;2(1):26-29.

13. Bitterling H, Rock C, Reiser M. [Computed tomography in the diagnosis of inflammatory bowel disease -methodology of MSCT and clinical results]. Radiologe. 2003;43(1):17-25.

14. Hussain SA, Esposito SP, Rubin M. Identification of small bowel diverticula with double-balloon enteroscopy following non-diagnostic capsule endoscopy. Dig Dis Sci. 2009;54(10):2296-2297.

15. Miehlke S, Tausche AK, Bruckner S, Aust D, Morgner A, Madisch A. Retrieval of two retained endoscopy capsules with retrograde double-balloon enteroscopy in a patient with a history of complicated small-bowel disease. Endoscopy. 2007;39(Suppl 1):E157.

16. Liu CH, Huang KW, Mo YH, Yang PM. Enterolith ileus in a patient with jejunal diverticulosis: sonographic findings. J Clin Ultrasound. 2007;35(3):169-173.

17. Yaqub S, Evensen BV, Kjellevold K. Massive rectal bleeding from acquired jejunal diverticula. World $\mathrm{J}$ 
Emerg Surg. 2011;6:17.

18. Butler JS, Collins CG, McEntee GP. Perforated jejunal diverticula: a case report. J Med Case Rep. 2010;4:172.

19. Wilcox RD, Shatney CH. Surgical significance of acquired ileal diverticulosis. Am Surg. 1990;56(4):222-225.

20. Rockey DC: Occult gastrointestinal bleeding. In Current Diagnosis \& Treatment in Gastrenterology. Edited by Friedman SL, McQuiad KR, Grendell JH. McGrawHill; 2003:83-95.

21. Novac JS, Tobias J, Barkin JS: Non surgical management of acute jejunal diverticulitis: A review. Am J Gastrenterol 1997, 92:1929-1931.

22. Herrington JL, Jr. Perforation of acuired diverticula of the jejunum and ileum. Analysis of reported cases. Surgery. 1962;51:426-433.

23. Albu E, Parikh V, Alankar S, Gerst PH. Perforated solitary jejunal diverticulum. South Med J. 1995;88(5):575576.
24. Falidas E, Vlachos K, Mathioulakis S, Archontovasilis F, Villias C. Multiple giant diverticula of the jejunum causing intestinal obstruction: report of a case and review of the literature. World J Emerg Surg. 2011;6(1):8.

25. Matteoni R, Lolli E, Barbieri A, D'Ambrosi M. Perforated jejunal diverticulitis: personal experience and diagnostic with therapeutical considerations. Ann Ital Chir. 2000;71(1):95-98.

26. Alvarez J, Jr., Dolph J, Shetty J, Marjani M. Recurrent rupture of jejunal diverticula. Conn Med. 1982;46(7):376-378.

27. Garg N, Khullar R, Sharma A, Soni V, Baijal M, Chowbey P. Total laparoscopic management of large complicated jejunal diverticulum. J Minim Access Surg. 2009;5(4):115-117.

28. Cross MJ, Snyder SK. Laparoscopic-directed small bowel resection for jejunal diverticulitis with perforation. J Laparoendosc Surg. 1993;3(1):47-49. 